\title{
Dilute Solution of Bisphenol A Polycarbonate
}

\author{
Takuji Tsuji, Takashi Norisuye, and Hiroshi Fujita \\ Department of Polymer Science, Osaka University, \\ Toyonaka 560, Japan.
}

(Received May 1, 1975)

\begin{abstract}
Narrow-distribution fractions of bisphenol A polycarbonate in tetrahydrofuran (THF) and chloroform were studied by light-scattering, sedimentation velocity, and viscosity measurements over the range of molecular weights from $4 \times 10^{3}$ to $5 \times 10^{5}$. The asymptotic values of a dimensionless quantity $A_{2} \bar{M}_{w} /[\eta]$ and the interpenetration function $\Psi$ where significantly lower than those usually expected for flexible polymers, suggesting a certain stiffness of the polycarbonate chain. Here $A_{2}$ is the second virial coefficient, $\bar{M}_{w}$ is the weight-average molecular weight, and $[\eta]$ is the intrinsic viscosity. Data for statistical radii $\left\langle S^{2}\right\rangle^{1 / 2}$, sedimentation coefficients $s_{0}$, and [ $\left.\eta\right]$ in THF were analyzed in terms of the wormlike chain model. First, the three parameters $q, M_{L}$, and $d$ characterizing the wormlike cylinder were estimated by using a recent theory of $s_{0}$ by Yamakawa and Fujii and assuming that the hydrodynamic volume per gram is equal to the partial specific volume of the polymer. Here $q$ is the persistence length, $M_{L}$ is the shift factor defined as the molar weight per contour length, and $d$ is the diameter of the cylinder. The results were $q=18 \mathrm{~A}, M_{L}=26$ daltons/A, and $d=6.6 \mathrm{~A}$. It was then shown that these molecular parameters and small expansion factors for the excluded volume allowed a consistent interpretation of the experimental data for $s_{0},\left\langle S^{2}\right\rangle$, and $[\eta]$. The consistency between the intra- and inter-molecular excluded-volume effects were also examined by use of the perturbation theories for $\alpha_{s}$ (expansion factor for $\left.\left\langle S^{2}\right\rangle\right)$ and for $A_{2}$ of the wormlike beads. An important finding from the present study is that, while $\alpha_{s}$ of the polycarbonate in THF is rather small, the binary cluster integral between a pair of monomers is quite large (about $170 \times 10^{-24} \mathrm{~cm}^{3}$ ).
\end{abstract}

KEY WORDS Bisphenol A polycarbonate / Flexibility / Wormlike

Chain / Persistence Length / Dilute Solution / Light Scattering /

Sedimentation Coefficient / Expansion Factor / Second Virial Coefficient /

It has become apparent in recent years that the behavior of such macromolecules as cellulose nitrate, ${ }^{1,2}$ polyisocynates, ${ }^{3}$ and amylose ${ }^{4}$ in dilute solution is not described adequately by the twoparameter theory $y^{5,6}$ and that this fact is primarily ascribable to the stiff or semiflexible nature of their molecular chains. Typical observations with these polymers are that the exponent $\nu$ for the Houwink-Mark-Sakurada equation is higher than 0.8 , often approaching unity, but the intramolecular excluded-volume effect is rather small.

Long ago, Schulz and Horbach ${ }^{7}$ remarked that bisphenol A polycarbonate, hereafter referred to as polycarbonate, belonged, in qualitative terms, to an intermediate class between flexible and semiflexible chains, since they found a value of 0.82 for $\nu$ in dichloromethane $(D C M)$. Note that this $\nu$ value is a little higher than the limiting value of 0.8 for flexible chains with extremely large excluded volumes. ${ }^{5}$ Subsequently, similar values of $\nu$ have been reported for polycarbonate: $0.80-0.82$ in $\mathrm{DCM},{ }^{8-10} 0.82$ in chloroform $\left(\mathrm{CHCl}_{3}\right),{ }^{8,10} 0.82$ in tetrachloroethane, ${ }^{8}$ and $0.76-0.78$ in ethylene dichloride (EDC). ${ }^{8,10}$ These results raise the interesting question of whether polycarbonate molecules are flexible or stiff. There have been a few comments ${ }^{8,9,11}$ on the flexibility of polycarbonate. For example, Yamakawa, ${ }^{11}$ analyzing the data of Schulz-Horbach ${ }^{7}$ for molecular weights $\bar{M}_{\mathrm{SD}}$ from sedimentation and diffusion coefficients, intrinsic viscosities $[\eta]$, and second virial coefficients $A_{2}$ for DCM and tetrahydro- 
furan (THF) solutions, found for a dimensionless quantity $A_{2} \bar{M}_{w} /[\eta]$ values that were significantly lower than those of flexible polymers in good solvents. He then proposed to introduce a parameter characterizing the chain stiffness for interpretation of this fact. On the other hand, Berry, et al., ${ }^{9}$ concluded from viscosity and light-scattering measurements that polycarbonate in DCM was flexible and very expanded due to an unusually large excluded-volume effect.

The present work has been undertaken to find an answer to these conflicting opinions on the flexibility of polycarbonate in dilute solution. For this purpose, careful measurements of light scattering, sedimentation velocity, osmotic pressure, and viscosity were made on sharply fractionated samples, ranging in molecular weight from $4 \times 10^{3}$ to $5 \times 10^{5}$, in two solvents, THF and $\mathrm{CHCl}_{3}$.

\section{EXPERIMENTAL}

\section{Samples}

Four samples of polycarbonate, A (viscosityaverage molecular weight $\left.\bar{M}_{v}=26 \times 10^{4}, 70 \mathrm{~g}\right), \mathrm{C}$ $\left(\bar{M}_{v}=9.3 \times 10^{4}, \quad 50 \mathrm{~g}\right), \quad \mathrm{T} \quad\left(\bar{M}_{v}=2.5 \times 10^{4}, 20 \mathrm{~g}\right)$, and $\mathrm{S}\left(\bar{M}_{v}=1 \times 10^{4}, 20 \mathrm{~g}\right)$, supplied by Mitsubishi Gas Chemical Co., Ltd., were treated in the manner described below. First, samples A and $\mathrm{C}$ were each divided into two parts by fractional precipitation with DCM as a solvent and isopropanol as a precipitant. The first fraction $\left(\bar{M}_{v}=30 \times 10^{4}, 50 \mathrm{~g}\right)$ obtained from $\mathrm{A}$ and a mixture of the second fraction $\left(\bar{M}_{v}=11.5 \times 10^{4}\right.$, $20 \mathrm{~g}$ ) from $\mathrm{A}$ and the first one $\left(\bar{M}_{v}=12 \times 10^{4}\right.$, $27 \mathrm{~g})$ from $C$ are designated $F$ and $D$, respectively. Samples F, D, T, and $\mathrm{S}$ were then each divided into a number of fractions by repeated fractional precipitation, in which the DCM$n$-heptane mixture of an appropriate composition was slowly added onto a DCM solution of polymer until the solution became turbid. In this way, almost the entire amount of the dissolved polymer was recovered without crystallization. Fractions of similar molecular weight were combined, and each of the combined fractions was refractionated, using the same solvent-precipitant system as above. In this way, 21 fractions were obtained from F, 16 fractions from $D, 11$ fractions from $T$, and 6 fractions from $S$. They were reprecipitated from DCM solutions into isopropanol and vacuumdried for two days at room temperature. From these fractions we selected 12 fractions for the present work and stored them in nitrogen-filled bottles at $-20^{\circ} \mathrm{C}$.

\section{Osmometry}

Measurements were made on a Mechrolab 502 high-speed membrane osmometer or a Knauer membrane osmometer fitted with a Sartorius Ultracellafilter membrane. EDC of 20 or $30^{\circ} \mathrm{C}$ was used as the solvent.

\section{Light-Scattering Measurements}

Apparatus and Calibration. Use was made of a Fica 50 automatic light-scattering photometer after the original temperature-control system had been replaced by a contact thermo-regulator (Model MC-S1, Mitamura Riken Industrial Co.), which was inserted directly into the bath filled with filtered xylene. The heater in the bath was connected to a voltstat so as to adjust its capacity. These improvements allowed the solution in the cell to be regulated to within $\pm 0.01^{\circ} \mathrm{C}$, eliminating the heat from the light source.

The circular uniformity of the cells (five cylindrical cells) was checked at $25^{\circ} \mathrm{C}$ with a dilute aqueous solution of fluorescein (about $\left.10^{-6} \mathrm{~mol} / \mathrm{l}\right)$ filtered through a Millipore filter (Type PHWP 04700). Vertically polarized light of $436 \mathrm{~nm}$ wavelength was used for the excitation. Deviations of the normalized intensity $\left(I_{\theta} / I_{90}\right) \sin \theta$ from unity were found to be within $\pm 0.5 \%$ for all the cells over the angular range $15^{\circ}-150^{\circ}$. Here $I_{\theta}$ denotes the intensity of scattered light at an angle of $\theta$ relative to that of incident light. Therefore, no further volume correction was made. The angular dependence of the scattered light from pure benzene was examined with the same incident light to check our procedure for optical purification and to estimate the amount of stray light. Values of $\left(I_{\theta} / I_{90}\right) \sin \theta$ for benzene did not deviate from unity more than $\pm 0.5 \%$ at the scattering angles between 15 and $150^{\circ}$, thus checking the successful purification and the substantial absence of stray light. The constant of the instrument was determined by taking the Rayleigh ratios of 
benzene at $25^{\circ} \mathrm{C}$ for 436 and $546 \mathrm{~nm}$ to be $46.5 \times 10^{-6}$ and $16.1 \times 10^{-6}$, respectively. ${ }^{12}$ Pure benzene of $25^{\circ} \mathrm{C}$ was also examined at the scattering angle of $90^{\circ}$ with vertically or horizontally polarized incident light and unpolarized incident light of $436 \mathrm{~nm}$. In each case, the analyzer was set in the vertical or the holizontal direction or was removed. From these measurements the depolarization ratio $\rho_{u}$ was calculated by taking into account the transmittances of the polarizer and analyzer and the ratio of the sensitivities of the receiver optical system to horizontally and vertically polarized light. The values of $\rho_{u}$ thus obtained were in the range between 0.429 and 0.451 , depending on the realignment of the optical axis needed whenever the mercury lamp was exchanged. These $\rho_{u}$ values are in agreement with the reported values. ${ }^{13}$

Measurements on Polymer Solutions. Measurements on polycarbonate solutions were made at $25^{\circ} \mathrm{C}$ in the angular range from 30 to $150^{\circ}$, using, in most cases, vertically polarized incident light of the wavelength $436 \mathrm{~nm}$. Test solutions and solvents $\left(\mathrm{CHCl}_{3}\right.$ and $\left.\mathrm{THF}\right)$ were made free of dust and microgels by centrifugation at $2 \times 10^{4}-4 \times 10^{4} \mathrm{~g}$ for $1-2 \mathrm{hr}$ either in a Marusan (Type $50 \mathrm{~V}-\mathrm{S}$ ) or a Sorvall (Type RC2-B) centrifuge. The observed data were analyzed by a Berry plot, ${ }^{14}$ i.e., by taking the square-root of $K c / R_{\theta}$ on the ordinate, to evaluate $\bar{M}_{w}, A_{2}$, and $\left\langle S^{2}\right\rangle$. Here $K$ is the light-scattering constant of the system, $R_{\theta}$ is the reduced intensity of scattered light, $c$ is the $c$-scale concentration, $\bar{M}_{w}$ is the weight-average molecular weight, and $\left\langle S^{2}\right\rangle$ is the mean-square radius of gyration, all for the polymer solute.

It was not always easy to make THF solutions free of microgels. In fact, higher-molecular weight fractions were less soluble in THF than in such halogenated hydrocarbons as $\mathrm{CHCl}_{3}$, DCM, and EDC. These THF solutions were therefore filtered through a sintered glass filter, and heated at $50^{\circ} \mathrm{C}$ for $1-2 \mathrm{hr}$, followed by centrifugation for $2-5 \mathrm{hr}$. Their concentrations were checked by measuring the flow times in a viscometer and corrected when necessary. The corrections were at most 2-3\%. At lower angles, the intensities of scattered light from THF solutions so purified were still higher than expected, probably due to the presence of still unremoved microgels. The data in THF at 30, 37.5 , and $45^{\circ}$ were therefore discarded in the data analyses.

Specific Refractive Index Increment $(d n / d c)$. The values of $(d n / d c)$ of polycarbonate in THF, $\mathrm{CHCl}_{3}$, and EDC at $25^{\circ} \mathrm{C}$, determined by use of a differential refractometer of Schulz-Cantow type, were $0.167\left(\mathrm{~cm}^{3} / \mathrm{g}\right)$ at $436 \mathrm{~nm}$ and 0.155 at $546 \mathrm{~nm}$ in $\mathrm{CHCl}_{3}, 0.194$ at $436 \mathrm{~nm}$ and 0.182 at $546 \mathrm{~nm}$ in THF, and 0.166 at $436 \mathrm{~nm}$ and 0.154 at $546 \mathrm{~nm}$ in EDC. The values in $\mathrm{CHCl}_{3}$ may be compared to the reported values, $0.164\left(20^{\circ} \mathrm{C}\right.$, $436 \mathrm{~nm})^{15}$ and $0.156\left(30^{\circ} \mathrm{C}, 546 \mathrm{~nm}\right) .{ }^{10}$ Furthermore, the values obtained with THF are in agreement with the data of Schulz-Horbach, ${ }^{7}$ $0.194(436 \mathrm{~nm})$ and $0.182(546 \mathrm{~nm})$ at $27^{\circ} \mathrm{C}$.

\section{Viscometry}

Viscosity measurements were made on $\mathrm{CHCl}_{3}$, THF, and DCM solutions at $25^{\circ} \mathrm{C}$. The viscometers used were of the Ubbelohde suspended-level type designed to have flow times of about $200 \mathrm{sec}$ for the solvents. Neither the kinetic-energy nor the shear-rate correction was necessary under any experimental conditions examined. The Huggins plot $^{16}$ and the MeadFuoss plot $^{17}$ were combined to determine $[\eta]$.

\section{Ultracentrifugation}

Sedimentation Velocity. Ultracentrifugation of polycarbonate fractions in $\mathrm{THF}$ at $25^{\circ} \mathrm{C}$ was carried out by use of a Beckman-Spinco Model E ultracentrifuge equipped with an electronic speed-control unit. An aluminum single-sector cell of $12 \mathrm{~mm}$ in depth was used. The rotor was spun at 59,700 and 47,800 rpm. Schlieren boundary curves were photographed on the Fuji spectroscopic plates and were read with the aid of a Nikon Shadowgraph Model 6. The usual peak method was used to determine the sedimentation coefficient $s$ for each initial concentration. For the two lowest molecular weight fractions S-3 and S-4, however, the Baldwin method $^{18}$ was employed, since freely sedimenting boundary curves were not obtained for their solutions. In all cases studied, plots of $\ln r_{\mathrm{m}}$ (or $\ln c_{\mathrm{p}}$ in the case of the Baldwin method) against time $t$ were convex downward, indicating the existencc of a pressure effect on $s .{ }^{19}$ Here $r_{\mathrm{m}}$ is the radial distance to the maximum 
gradient, $c_{\mathrm{p}}$ is the concentration in the plateau region, and $t$ is the time of centrifugation. It was found for typical fractions that the values of $s$ estimated from the initial slopes of $\ln r_{\mathrm{m}}$ vs. $t$ did not differ more than about $1 \%$ from those corrected for the pressure effect by the Blair-Williams method. ${ }^{20}$ Therefore, the desired values of $s$ for all of the fractions were estimated from the initial slopes of the $\ln r_{\mathrm{m}}$ vs. $t$ plots (or the $\ln c_{\mathrm{p}} v s . t$ plots). The concentration $c_{\mathrm{p}}$ of the plateau region was determined from the observed gradient curves by using the calibration constants determined previously. ${ }^{21}$

Sedimentation Equilibrium. The values of $\bar{M}_{w}$ for fractions $\mathrm{T}-8, \mathrm{~S}-3$, and $\mathrm{S}-4$ were determined by sedimentation equilibrium, since the lightscattering method seemed less adequate for these samples of low molecular weight. A Kel-F 12 $\mathrm{mm}$ double sector cell was used. The length of the solution column was adjusted to $2.5 \mathrm{~mm}$ as usual. The rotor speeds were in the range from 18,000 to $30,000 \mathrm{rpm}$, depending on the molecular weight. The partial specific volume of the polymer in THF at $25^{\circ} \mathrm{C}$ was determined by using a bicapillary-type pycnometer. The

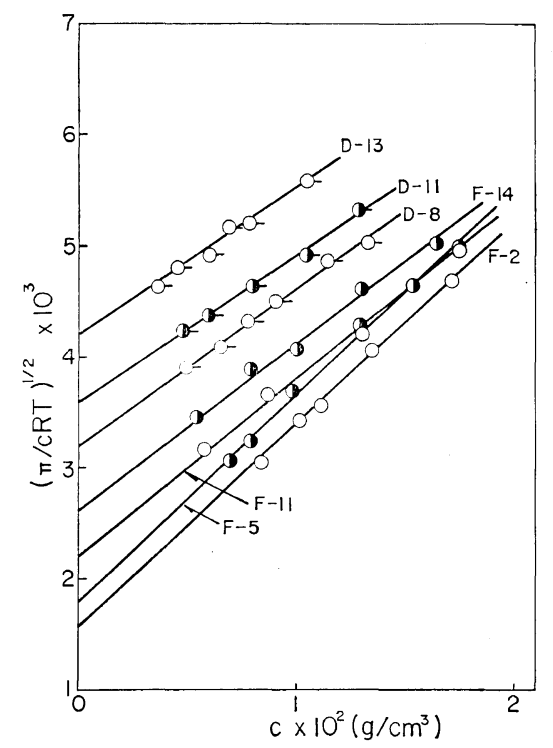

Figure 1. Square root plots of reduced osmotic pressure $\pi /(c R T)$ vs. polymer concentration $c$ for polycarbonate fractions in EDC at $20^{\circ} \mathrm{C}$ (horizontal pip) and $30^{\circ} \mathrm{C}$ (no pip). result, $0.774\left(\mathrm{~cm}^{3} / \mathrm{g}\right)$, agreed with the value of 0.7738 (at $20^{\circ} \mathrm{C}$ ) reported by Schulz-Horbach. ${ }^{7}$

\section{RESULTS}

\section{Molecular Weight Determinations}

Figure 1 shows osmotic pressure data on polycarbonate fractions in EDC. Values of the number-average molecular weights $\bar{M}_{n}$ and $A_{2}$ obtained from this graph are summarized in Table I. Figure 2 illustrates light-scattering data in $\mathrm{CHCl}_{3}$. The data shown refer to vertically polarized incident light of $436 \mathrm{~nm}$. It is to be observed that the data points for fractions F-2 and $\mathrm{F}-5$ at higher concentrations deviate from the indicated straight lines. These derivations may be attributable to an appreciable nonideality of the systems (see $A_{2}$ values in Table $\mathrm{I}$ ).

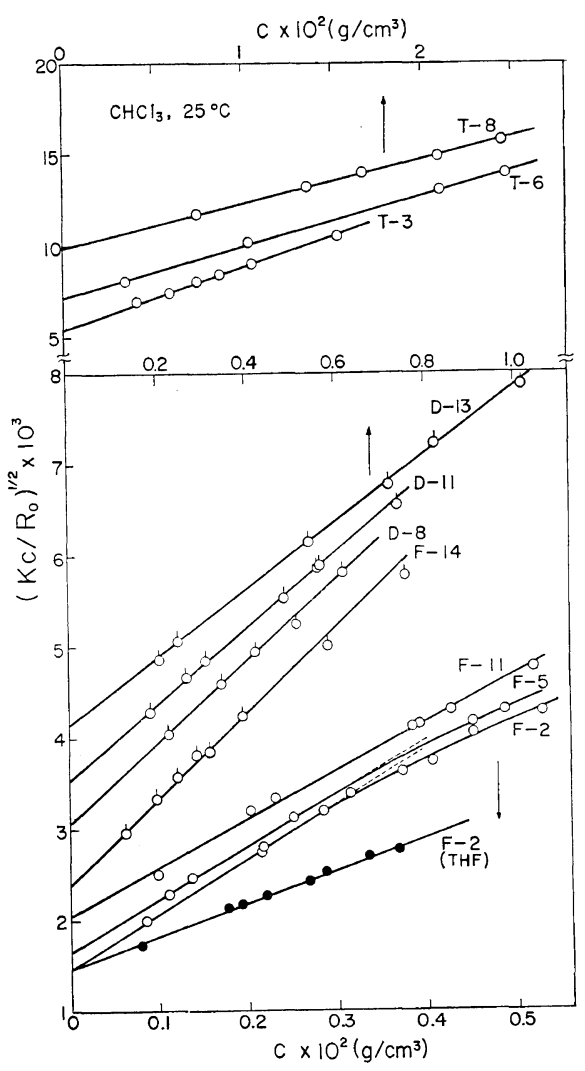

Figure 2. Plots of $\left(K c / R_{0}\right)^{1 / 2}$, values of $\left(K c / R_{\theta}\right)^{1 / 2}$ at zero angle of scattering, vs. $c$ for polycarbonate fractions in $\mathrm{CHCl}_{3}(\mathrm{O})$ and $\operatorname{THE}(\bullet)$ at $25^{\circ} \mathrm{C}$. 
Table I. Results from osmotic pressure and light-scattering measurements on polycarbonate fractions

\begin{tabular}{|c|c|c|c|c|c|c|c|}
\hline \multirow{2}{*}{ Fraction } & \multicolumn{2}{|c|}{$\begin{array}{l}\text { Osmotic pressure, } \\
\text { in EDC at } 30^{\circ} \mathrm{C}\end{array}$} & \multicolumn{4}{|c|}{ Light scattering } & \multirow{2}{*}{$\bar{M}_{w} / \bar{M}_{n}^{\mathrm{e}}$} \\
\hline & $\bar{M}_{n} \times 10^{-4}$ & $\begin{array}{c}A_{2} \times 10^{4} \\
\mathrm{~cm}^{3} \mathrm{~mol} / \mathrm{g}^{2}\end{array}$ & $\begin{array}{l}\text { Solvent, } \\
25^{\circ} \mathrm{C}\end{array}$ & $\bar{M}_{w} \times 10^{-4}$ & $\begin{array}{c}A_{2} \times 10^{4} \\
\mathrm{~cm}^{3} \mathrm{~mol} / \mathrm{g}^{2}\end{array}$ & $\stackrel{\left\langle S^{2}\right\rangle^{1 / 2}}{\mathrm{~A}}$ & \\
\hline \multirow[t]{2}{*}{$\mathrm{F}-2$} & 40.1 & 5.75 & $\mathrm{CHCl}_{3}$ & 46.3 & 8.91 & 424 & 1.16 \\
\hline & & & THF & 46.9 & 5.23 & 378 & \\
\hline \multirow[t]{3}{*}{ F-5 } & 30.9 & 6.67 & $\mathrm{CHCl}_{3}$ & 36.7 & 9.65 & 364 & 1.18 \\
\hline & & & THF & 37.2 & 5.97 & 322 & \\
\hline & & & EDC & 35.9 & 7.43 & 360 & \\
\hline \multirow[t]{4}{*}{$F-11$} & 20.5 & 7.04 & $\mathrm{CHCl}_{3}$ & 23.3 & 10.8 & 282 & 1.14 \\
\hline & & & $\mathrm{CHCl}_{3}{ }^{\mathrm{b}}$ & 23.3 & 10.8 & 278 & \\
\hline & & & $\mathrm{CHCl}_{3}{ }^{\mathrm{c}}$ & 22.9 & 10.7 & 276 & \\
\hline & & & THF & 23.6 & 5.91 & 255 & \\
\hline \multirow[t]{2}{*}{ F-14 } & 14.6 & 7.83 & $\mathrm{CHCl}_{3}$ & 17.7 & 11.3 & 245 & 1.23 \\
\hline & & & THF & 18.1 & 7.01 & 214 & \\
\hline \multirow[t]{3}{*}{ D-8 } & 9.77 & $9.00^{\mathrm{a}}$ & $\mathrm{CHCl}_{3}$ & 10.6 & 13.6 & 187 & 1.11 \\
\hline & & & $\mathrm{CHCl}_{3} \mathrm{~b}$ & 10.6 & 13.9 & 189 & \\
\hline & & & THF & 11.1 & 7.95 & 170 & \\
\hline \multirow[t]{2}{*}{ D-11 } & 7.72 & $9.50^{\mathrm{a}}$ & $\mathrm{CHCl}_{3}$ & 8.00 & 14.7 & 161 & 1.06 \\
\hline & & & THF & 8.35 & 8.42 & 144 & \\
\hline \multirow[t]{2}{*}{ D-13 } & 5.64 & $11.0^{\mathrm{a}}$ & $\mathrm{CHCl}_{3}$ & 5.81 & 15.4 & 141 & 1.04 \\
\hline & & & THF & 5.92 & 8.70 & - & \\
\hline \multirow[t]{3}{*}{$\mathrm{T}-3$} & - & - & $\mathrm{CHCl}_{3}$ & 3.37 & 18.6 & - & - \\
\hline & & & $\mathrm{CHCl}_{3}{ }^{\mathrm{b}}$ & 3.38 & 18.6 & - & \\
\hline & & & $\mathrm{CHCl}_{3} \mathrm{c}$ & 3.48 & 18.2 & - & \\
\hline \multirow[t]{3}{*}{$\mathrm{T}-6$} & - & - & $\mathrm{CHCl}_{3}$ & 1.93 & 20.1 & - & - \\
\hline & & & $\mathrm{CHCl}_{3} \mathrm{~b}$ & 1.93 & 19.9 & - & \\
\hline & & & $\mathrm{CHCl}_{3} \mathrm{C}$ & 1.94 & 19.3 & - & \\
\hline \multirow{4}{*}{$\mathrm{T}-8$} & - & - & $\mathrm{CHCl}_{3}$ & 1.02 & 24.0 & - & - \\
\hline & & & $\mathrm{CHCl}_{3} \mathrm{~b}$ & 1.02 & 23.8 & - & \\
\hline & & & $\mathrm{CHCl}_{3} \mathrm{c}$ & 1.04 & 23.5 & - & \\
\hline & & & $\mathrm{THF}^{\mathrm{d}}$ & 1.07 & - & & \\
\hline S-3 & - & - & $\mathrm{THF}^{\mathrm{d}}$ & 0.63 & - & & - \\
\hline S-4 & - & - & $\mathrm{THF}^{\mathrm{d}}$ & 0.43 & - & & - \\
\hline
\end{tabular}

a Measured at $20^{\circ} \mathrm{C}$.

b Measured with unpolarized incident light of $436 \mathrm{~nm}$ wavelength.

c Measured with unpolarized incident light of $546 \mathrm{~nm}$ wavelength.

d Determined by sedimentation equilibrium.

e Average of $\bar{M}_{w}$ determined under different experimental conditions.

Table I also summarizes the values of $\bar{M}_{w}$ and $A_{2}$ obtained from light-scattering and sedimentation equilibrium measurements. It is seen that the values of $\bar{M}_{w}$ determined in different solvents agree with each other within the accuracy of the light-scattering experiments, and also that the values of $\bar{M}_{w}$ and $A_{2}$ obtained for different incident beams are in close agreement with one another. Furthermore, the $\bar{M}_{w}$ value for fraction T-8 from light scattering agrees closely with that from ultracentrifugation. It is to be noted that the values of $A_{2}$ for all the systems studied are unusually large.

The eighth column of Table I, which gives the ratios of $\bar{M}_{w}$ to $\bar{M}_{n}$, shows that the samples used were fairly narrow in molecular weight distribution. In fact, the GPC curves taken with THF substantiated this conclusion. 


\section{Mean-Square Radius of Gyration}

Figure 3 shows plots of $\left(\bar{M}_{w} K c / R_{\theta}\right)_{\mathrm{c}=0}^{1 / 2} v s$. $\sin ^{2}(\theta / 2)$ for polycabonate fractions in $\mathrm{CHCl}_{3}$ and THF. The plotted points for each fraction in $\mathrm{CHCl}_{3}$ follow a straight line over the entire

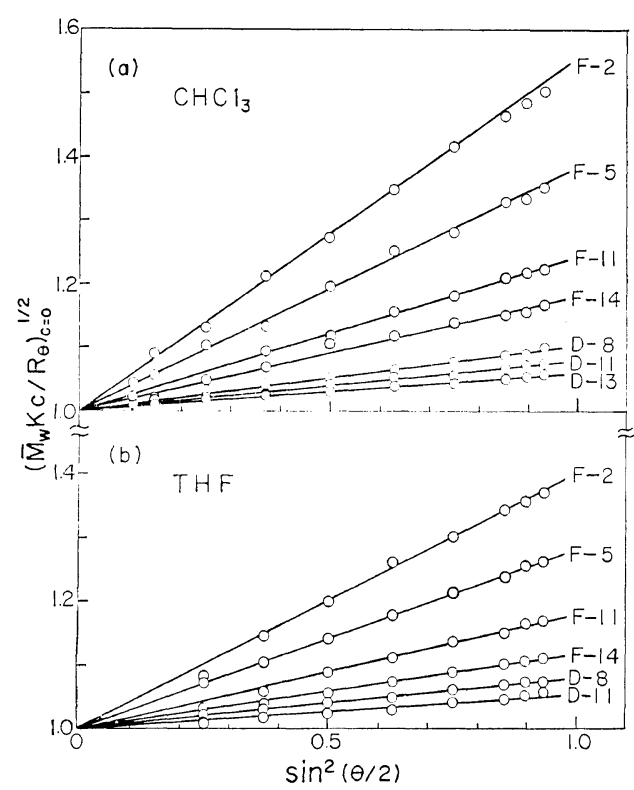

Figure 3. Angular dependence of $\left(\bar{M}_{w} K c / R_{\theta}\right)_{\mathrm{c}=0^{1 / 2}}$ for polycarbonate fractions in $\mathrm{CHCl}_{3}$ (a) and THF (b) at $25^{\circ} \mathrm{C}$.

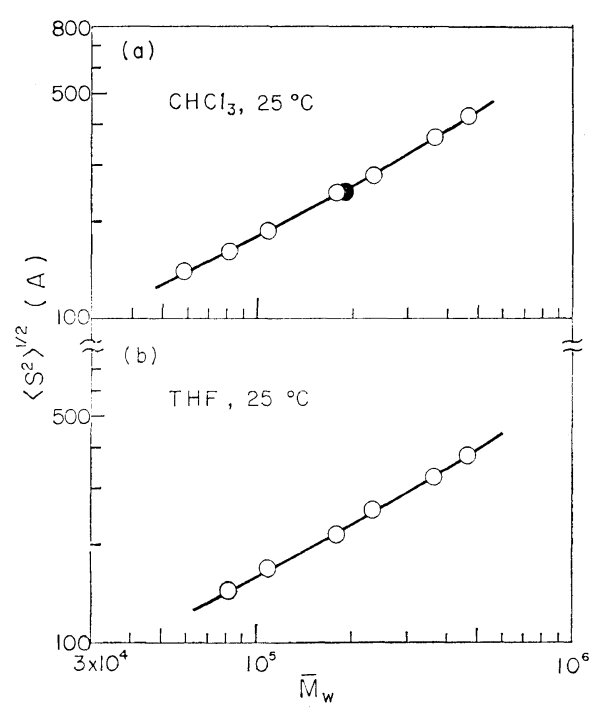

Figure 4. Molecular weight dependence of $\left\langle S^{2}\right\rangle^{1 / 2}$ for polycarbonate in $\mathrm{CHCl}_{3}$ (a) and THF (b): $\bigcirc$, this work; $\bullet$, Berry, et al. ${ }^{9}$ angular range studied. Data in THF at lower angles were omitted for the reason mentioned before. Probably the values of $\left\langle S^{2}\right\rangle$ determined in this solvent were less accurate than those in $\mathrm{CHCl}_{3}$. Numerical results of $\left\langle S^{2}\right\rangle^{1 / 2}$ are given in the seventh column of Table I. The values of $\left\langle S^{2}\right\rangle^{1 / 2}$ obtained for different incident lights agree well with one another. Molecular weight dependences of $\left\langle S^{2}\right\rangle^{1 / 2}$ in $\mathrm{CHCl}_{3}$ and THF are depicted in Figure 4. Our values of $\left\langle S^{2}\right\rangle^{1 / 2}$ in $\mathrm{CHCl}_{3}$ are consistent with the datum of Berry, et al., ${ }^{9}$ indicated by the filled circle. The $\left\langle S^{2}\right\rangle^{1 / 2}$ data in both $\mathrm{CHCl}_{3}$ and THF appear to follow curves slightly concave upword. Their slopes are close to 0.5 for $\bar{M}_{w}$ up to $20 \times 10^{4}$ and then increase rather rapidly with $\bar{M}_{w}$. This trend may be interpreted as indicating that there are excluded-volume effects which are small in the range of low molecular weights but become appreciable with further increase in $\bar{M}_{w}$. Berry, et al., ${ }^{9}$ found a much larger molecular weight dependence of $\left\langle S^{2}\right\rangle^{1 / 2}$ in DCM and attributed it to a pronounced excluded-volume effect. However, since their data are confined to a range of relatively high molecular weights $\left(28 \times 10^{4}<\bar{M}_{w}<76 \times 10^{4}\right)$, no direct comparison

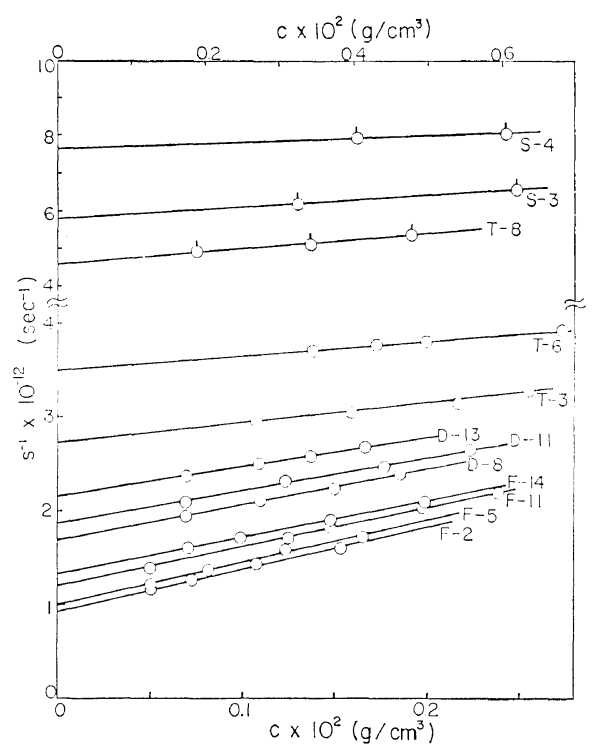

Figure 5. Dependence of sedimentation coefficient $s$ on initial concentration for polycarbonate fractions in $\mathrm{THF}$ at $25^{\circ} \mathrm{C}$ : $b$, for upper $c$-scale concentration. 
of them with our results is feasible. It should be remarked that the $\left\langle S^{2}\right\rangle^{1 / 2}$ data of Berry, et al., coupled with those of Schulz and Horbach ${ }^{7}$ for two samples of low molecular weight, give a molecular weight dependence compatible with our results for $\mathrm{CHCl}_{3}$ and THF.

\section{Sedimentation Coefficient}

Figure 5 shows the dependence of $s$ on initial concentration $c$ for polycarbonate fractions in THF. The data points for each fraction obey a linear relation, which allows the determination of the limiting sedimentation coefficient $s_{0}$ and $k_{\mathrm{s}}$. Here $k_{\mathrm{s}}$ is a constant defined by

$$
s^{-1}=s_{0}^{-1}\left(1+k_{\mathrm{s}} c\right)
$$

The values of $s_{0}$ and $k_{\mathrm{s}}$ thus determined are sum-

Table II. Results from sedimentation velocity and viscosity measurements on polycarbonate fractions

\begin{tabular}{|c|c|c|c|c|c|}
\hline \multirow{2}{*}{$\begin{array}{l}\text { Frac- } \\
\text { tion }\end{array}$} & \multirow{2}{*}{$\begin{array}{c}\begin{array}{c}s_{0} \times 10^{13} \\
\mathrm{sec}\end{array} \\
\mathrm{THF},\end{array}$} & \multirow{2}{*}{$\begin{array}{c}\begin{array}{c}k_{\mathrm{s}}, \\
\mathrm{d} l / \mathrm{g}\end{array} \\
5^{\circ} \mathrm{C}\end{array}$} & \multicolumn{3}{|c|}{$\mathrm{d} l / \mathrm{g}, 25^{\circ} \mathrm{C}$} \\
\hline & & & $\mathrm{CHCl}_{3}$ & DCM & THF \\
\hline F-2 & 10.8 & 4.79 & 4.78 & 4.45 & 3.93 \\
\hline F-5 & 10.0 & 4.50 & 4.08 & 3.88 & 3.47 \\
\hline F-11 & 8.33 & 3.50 & 2.79 & 2.70 & 2.42 \\
\hline F-14 & 7.52 & 2.93 & 2.35 & 2.20 & 2.01 \\
\hline D-8 & 5.95 & 2.26 & 1.56 & 1.50 & 1.39 \\
\hline D-11 & 5.35 & 1.87 & 1.28 & 1.22 & 1.09 \\
\hline D-13 & 4.65 & 1.49 & 1.02 & 0.970 & 0.914 \\
\hline $\mathrm{T}-3$ & 3.66 & 0.81 & 0.708 & 0.670 & 0.657 \\
\hline $\mathrm{T}-6$ & 2.86 & 0.45 & 0.485 & 0.470 & 0.462 \\
\hline $\mathrm{T}-8$ & 2.16 & 0.32 & 0.304 & 0.302 & 0.299 \\
\hline S-3 & 1.70 & - & 0.203 & 0.195 & 0.199 \\
\hline$S-4$ & 1.31 & - & 0.154 & 0.149 & 0.153 \\
\hline
\end{tabular}

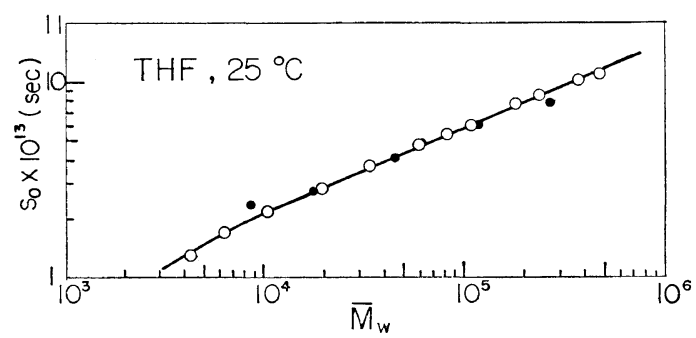

Figure 6. Molecular weight dependence of limiting sedimentation coefficient $s_{0}$ for polycarbonate in THF at $25^{\circ} \mathrm{C}$ : $\bigcirc$, present data; $\bigcirc$, SchulzHorbach's data ${ }^{7}$ (reduced to $25^{\circ} \mathrm{C}$, see text) with $\bar{M}_{\mathrm{SD}}$ taken as $\bar{M}_{w}$. marized in Table II. The molecular weight dependence of $s_{0}$ is shown in Figure 6, where Schulz-Horbach's data $^{7}$ (at $20^{\circ} \mathrm{C}$ ) reduced to $s_{0}$ at $25^{\circ} \mathrm{C}$ by

$$
s_{25}=s_{20}(1-\bar{v} \rho)_{25} \eta_{20} /(1-\bar{v} \rho)_{20} \eta_{25}
$$

are also plotted. Here $\eta$ and $\rho$ are the solvent viscosity and density, respectively, and the subscripts refer to temperature. Our data are consistent with those of Schulz-Horbach (excepting two points for their highest and lowest samples), and can be expressed by

$$
s_{0}=4.05 \times 10^{-15} \bar{M}_{w}{ }^{0.43} \quad\left(6 \times 10^{3}<\bar{M}_{w}<5 \times 10^{5}\right)
$$

\section{Intrinsic Viscosity}

Numerical results from viscosity measurements are summarized in Table II. Figure 7 shows conventional $\log -\log$ plots of $[\eta]$ vs. $\bar{M}_{w}$ for polycarbonate in $\mathrm{DCM}$ at $25^{\circ} \mathrm{C}$. For comparison, we have also plotted the data from previous investigators. ${ }^{7-10}$ When compared at the same molecular weights, our $[\eta]$ are in close agreement with those of Schulz-Horbach ${ }^{7}$ and Berry, et al., ${ }^{9}$ in the region of high molecular weights, whereas in the region of lower molecular weights our values are slightly larger than the reported values. The exponent $\nu$ for the HouwinkMark-Sakurada equation appears to increase slightly with increasing molecular weight: about 0.72 for lower molecular weights and 0.77 for higher molecular weights. The latter value is

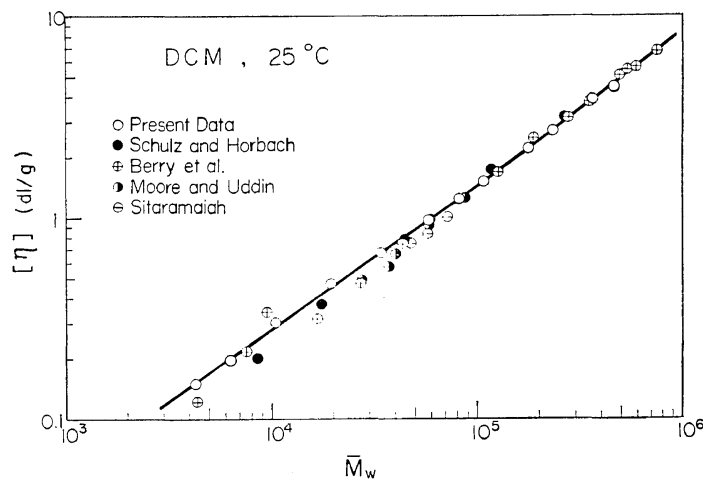

Figure 7. Log-log plots of $[\eta]$ vs. $\bar{M}_{w}$ for polycarbonate in DCM at $25^{\circ} \mathrm{C}$ : $\bigcirc$, this work; $\bigcirc$, Schulz and Horbach ${ }^{7}\left(\bar{M}_{\mathrm{SD}}\right.$ taken as $\left.\bar{M}_{w}\right) ; \ominus$, Sitaramaiah; $;^{8} \oplus$, Berry, et al. $;^{9} \bigcirc$, Moore and Uddin. ${ }^{10}$ 


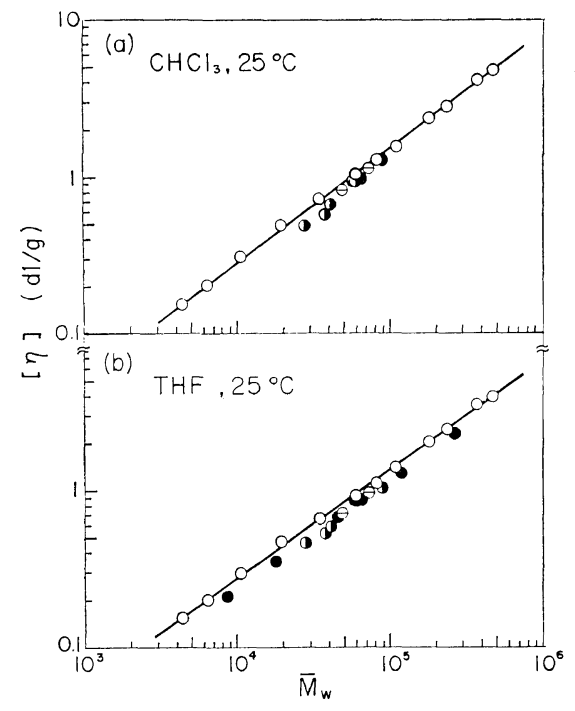

Figure 8. $\log -\log$ plots of $[\eta]$ vs. $\bar{M}_{w}$ for polycarbonate in $\mathrm{CHCl}_{3}$ (a) and THF (b) at $25^{\circ} \mathrm{C}$. The symbols are the same as in Figure 7.

smaller than the reported values, ${ }^{7-10} 0.80-0.82$. All these data for polycarbonate in DCM may be combined approximately by a relation

$$
[\eta]=2.99 \times 10^{-4} \bar{M}_{w}{ }^{0.74}, \mathrm{~d} l / \mathrm{g} \quad \text { (in DCM) }
$$

Figure 8 shows similar plots for the data in $\mathrm{CHCl}_{3}$ and THF. The plotted points are approximately represented by

$$
\begin{array}{ll}
{[\eta]=3.01 \times 10^{-4} \bar{M}_{w}{ }^{0.74}, \mathrm{~d} l / \mathrm{g}} & \left(\text { in } \mathrm{CHCl}_{3}\right) \\
{[\eta]=3.78 \times 10^{-4} \bar{M}_{w}{ }^{0.71}, \mathrm{~d} l / \mathrm{g}} & \text { (in } \mathrm{THF})
\end{array}
$$

Schulz-Horbach's values of $[\eta]$ in THF appear systematically below the line corresponding to eq 6. The discrepancy should not be due to errors in molecular weight determinations since, in the range of high molecular weights, their data in DCM agree with ours (see Figure 7).

The values of $\nu$ obtained for the three solvents are close to those reported for randomly coiled polymers in very good solvents, so that, as far as the $[\eta]-\bar{M}_{w}$ relationship is concerned, the polycarbonate molecule may be regarded as a random coil expanded with a large excludedvolume effect. However, the corresponding $\left\langle S^{2}\right\rangle$ data indicate that this effect is rather small. Similar contradictions have already been reported for cellulose nitrate $^{1,2}$ and amylose, ${ }^{4}$ both known as stiff chains. The present data for $\left\langle S^{2}\right\rangle$ and $[\eta]$ thus suggest that the polycarbonate chain in $\mathrm{CHCl}_{3}, \mathrm{THF}$, and DCM should have a certain stiffness.

\section{DISCUSSION}

\section{Comparison with Known Behavior of Flexible Chains}

To check the suggestion mentioned above we compare the data of $A_{2}$, [ $\left.\eta\right]$, and $\left\langle S^{2}\right\rangle$ for polycarbonate with available information on flexible chains. Figure 9 shows $A_{2} \bar{M}_{w} /[\eta]$ as a function of $[\eta] /[\eta]_{\theta}$ for polycarbonate in $\mathrm{CHCl}_{3}, \mathrm{THF}$, and DCM. Here $[\eta]_{\theta}$, the value of $[\eta]$ at the theta condition, has been estimated by using the relation $[\eta]_{\theta}=2.1 \times 10^{-3} \bar{M}_{w}{ }^{1 / 2}(\mathrm{~d} l / \mathrm{g})$ reported by Berry, et al., ${ }^{9}$ the plotted points for DCM have been calculated from the data of SchulzHorbach $^{7}$ and Berry, et al. ${ }^{9} \quad$ According to recent experimental studies of excluded-volume effects in flexible polymers, the relation between $A_{2} \bar{M}_{w} /[\eta]$ and $[\eta] /[\eta]_{\theta}$ for these polymers follows a universal curve, shown in Figure 9 by the thin line. ${ }^{5,6}$ Our values of $A_{2} \bar{M}_{w} /[\eta]$ appear significantly below this curve, being about 60 for THF and 90 for $\mathrm{CHCl}_{3}$. The data in DCM also exhibit a similar trend. Values for the

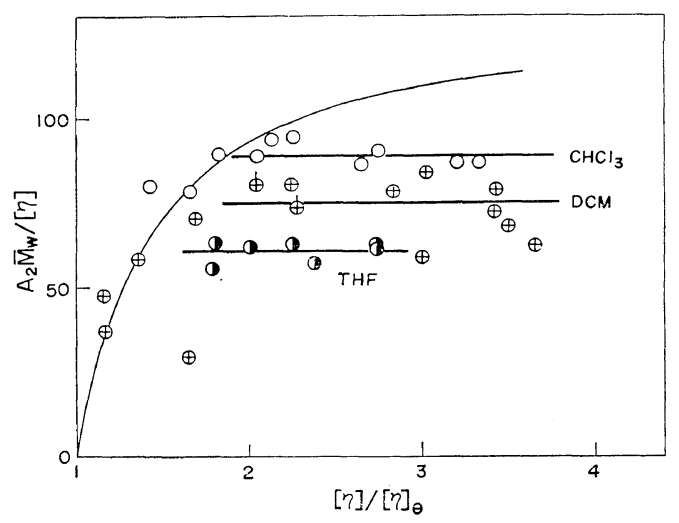

Figure 9. Dimensionless quantity $A_{2} \bar{M}_{w} /[\eta]$ plotted against $[\eta] /[\eta] \theta$ for polycarbonate in the indicated solvents. $[\eta]_{\theta}$, the value of $[\eta]$ under the theta condition, was estimated by using the relation $[\eta]_{\theta}=2.1 \times 10^{-3} \bar{M}_{w}^{1 / 2}, 9$ and the data for DCM were taken from ref $7(\oplus)$ and $9(\oplus)$. The thin line traces the typical data for flexible polymers. ${ }^{5,6}$ 
interpenetration function $\Psi$ defined by $A_{2} \bar{M}_{w}{ }^{2} /\left(4 \pi^{3 / 2} N_{\mathrm{A}}\left\langle S^{2}\right\rangle^{3 / 2}\right) \quad\left(N_{\mathrm{A}}\right.$ is the Avogadro number), though not shown here, are also lower (about 0.17 for THF and 0.19 for $\mathrm{CHCl}_{3}$ ) than the asymptotic value of $0.25-0.28$ known for flexible chains. ${ }^{5,6}$ These facts support the suggestion that polycarbonate chains in $\mathrm{CHCl}_{3}$, THF, and DCM may not be completely flexible but should have some stiffness. In the following, therefore, we attempt to examine whether the equilibrium and hydrodynamic data for polycarbonate can be interpreted by the wormlike chain, ${ }^{22}$ a typical model for stiff chains.

Data Analysis in Terms of The Wormlike Chain

According to the recent hydrodynamic theories by Yamakawa and Fujii, ${ }^{23,24} s_{0}$ and $[\eta]$ for an unperturbed wormlike chain (wormlike cylinder) are characterized by three parameters $q, M_{L}$, and $d$, where $q$ is the persistence length, $M_{L}$ is the shift factor defined as the ratio of the molecular weight $M$ to the contour length $L$, and $d$ is the diameter of the cylinder. Yamakawa and Fujii $^{24}$ proposed a method for determining these parameters from data of $s_{0}$ and $[\eta]$ and applied it successfully to DNA,

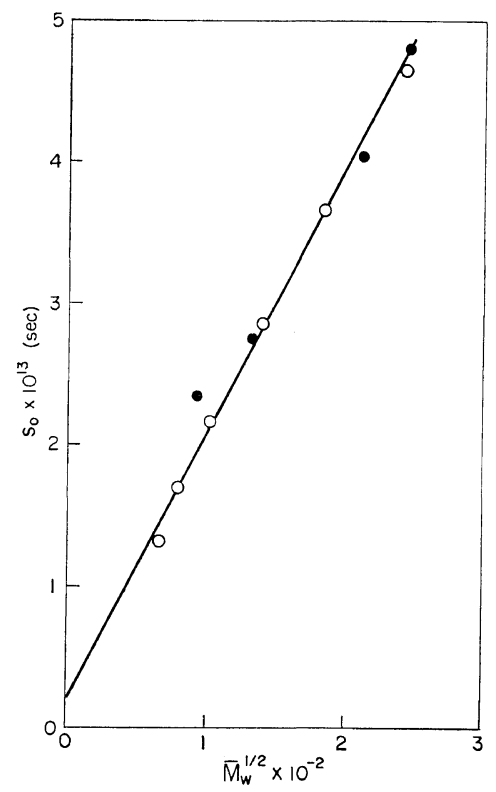

Figure 10. Plots of $s_{0}$ vs. $\bar{M}_{w}^{1 / 2}$ for polycabonate in $\mathrm{THF}$ at $25^{\circ} \mathrm{C}$. The symbols are the same as in Figure 6. poly(n-butyl isocynate), and cellulose nitrate. However, we have found that their method was ineffective for less stiff chains such as polycarbonate, and moreover, that the three parameters for this polymer were not determinable uniquely even if, in addition to the data for $s_{0}$ and $[\eta]$, those of $\left\langle S^{2}\right\rangle$ were available. For their evaluation, therefore, we have either to assume an appropriate value for one of them or to introduce a relation among them in advance. We here assume between $d$ and $M_{L}$ a relation

$$
d=\left(4 \bar{v} M_{L} / \pi N_{\mathrm{A}}\right)^{1 / 2}
$$

where $\bar{v}$ is the partial specific volume of the solute. The discussion to follow is restricted to the THF solutions because we have determined the set of data for $s_{0},[\eta]$, and $\left\langle S^{2}\right\rangle$ as functions of $\bar{M}_{w}$ only in THF.

Yamakawa-Fujii's expression ${ }^{23}$ for $s_{0}$ may be approximated by

$$
s_{0}=\frac{(1-\bar{v} \rho)}{3 \pi \eta_{0} N_{\mathrm{A}}}\left[1.843\left(M_{L} / 2 q\right)^{1 / 2} M^{1 / 2}+M_{L} f(d / 2 q)\right]
$$

if $L / q$ is sufficiently large and the excluded volume is negligible. Here $\eta_{0}$ is the solvent viscosity, and $f(d / 2 q)$, a function of $d / 2 q$, stands for the $A_{2}$ in the original paper. Equation 8 gives a linear plot of $s_{0} v s . M^{1 / 2}$, and its slope $S$ and ordinate intercept $I$, when combined with eq 7, allow $q, M_{L}$, and $d$ to be evaluated. Figure 10 shows such plots for polycarbonate in THF. Here the data have been shown only in the region of molecular weights for which the excluded-volume effect would be negligible. The straight line drawn gives $S=1.88 \times 10^{-15}$ $\left(\mathrm{sec} \cdot \mathrm{mol}^{1 / 2} / \mathrm{g}^{1 / 2}\right.$ ) and $I=0.20 \times 10^{-13}(\mathrm{sec})$. With the known values of $0.774\left(\mathrm{~cm}^{3} / \mathrm{g}\right)$ for $\bar{v}$ and $0.436 \times 10^{-2}$ (poise) for $\eta_{0}$, we obtain the values of $q, M_{L}$, and $d$, which are summarized in Table III together with the crystallographic data. $^{25}$ The values of 26 daltons/A for $M_{L}$ and

Table III. Wormlike-chain parameters

\begin{tabular}{|c|c|c|c|}
\hline & $q, \mathrm{~A}$ & $\begin{array}{c}M_{L}, \\
\text { daltons/A }\end{array}$ & $d, \mathrm{~A}$ \\
\hline & 18 & 26 & \\
\hline Crystallographic data 25 & - & 24.4 & $5.0-6.2$ \\
\hline
\end{tabular}
of polycarbonate

Polymer J., Vol. 7, No. 5, 1975 


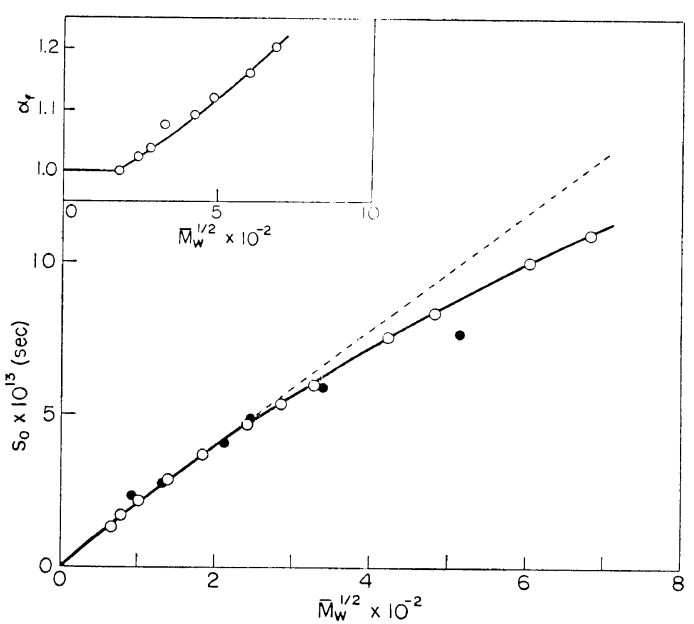

Figure 11. Plots of $s_{0} v s . \bar{M}_{w^{1 / 2}}$ for polycarbonate in THF at $25^{\circ} \mathrm{C}$. Solid line, best fit to the present data; dashed line, calculated from the YamakawaFujii theory ${ }^{23}$ for $s_{0}$ with the parameters given in Table III. The insert gives the friction-radius expansion factor $\alpha_{f}$ taken as the ratio of the calculated values (dashed line) to the observed values.

6.6 A for $d$ are in fair agreement with 24.4 and 5.0-6.2, respectively, obtained by Bonart ${ }^{25}$ from $\mathrm{X}$-ray analyses. However, this agreement should be accepted with reservation, since our results are based on the assumption of eq 7. The plots of $s_{0}$ vs. $\bar{M}_{w}^{1 / 2}$ are again depicted in Figure 11 together with the data for higher molecular weight fractions. The dashed line indicates the theoretical curve calculated by using the exact Yamakawa-Fujii expression with the parameters given in Table III. The line is linear with $\bar{M}_{w}^{1 / 2}$ in the range of $\bar{M}_{w}$ above $2.5 \times 10^{3}$ and indicates that the asymptotic expression, eq 8 , holds down to $\bar{M}_{w}{ }^{1 / 2} \approx 50$. However, the solid line fitting the data points deviates appreciably from it for $\bar{M}_{w}$ above $9 \times 10^{4}$. This may be ascribed to excludedvolume effects. The friction-radius expansion factor $\alpha_{f}$ taken as the ratio of the calculated $s_{0}$ (dashed line) to the observed values is shown in the insert of Figure 11. Interestingly, $\alpha_{f}$ increases approximately linearly with $\bar{M}_{w}{ }^{1 / 2}$ for $\bar{M}_{w}{ }^{1 / 2}>200$ but remains 1.2 even for the highest molecular weight fraction studied.

Next, we compare the experimental data for
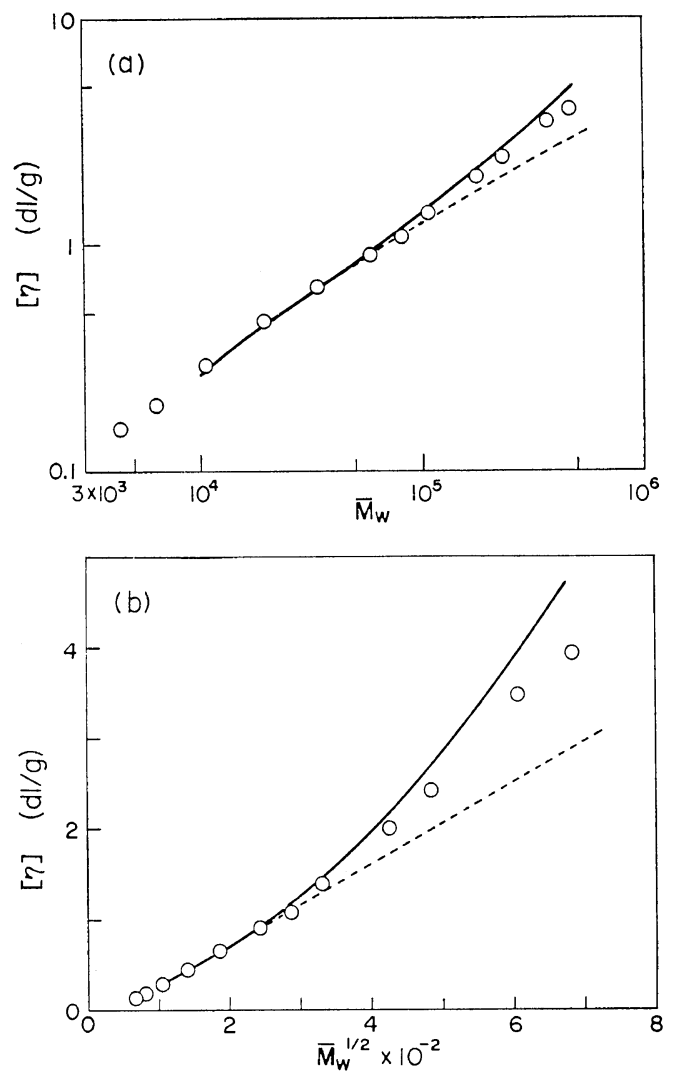

Figure 12. Comparison of experimental values of $[\eta]$ for polycarbonate in THF with theoretical ones from Yamakawa-Fujii's theory24 with the same

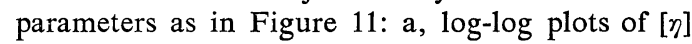
vs. $\bar{M}_{w} . \quad$ b, plots of $[\eta] v s . \bar{M}_{w}^{1 / 2}$. Dashed lines, for unperturbed chains; solid lines, values corrected for the excluded-volume effect with the $\alpha_{f}$ in Figure 11.

[ $\eta]$ and $\left\langle S^{2}\right\rangle$ in THF with the YamakawaFujii theory ${ }^{24}$ for the former and the BenoitDoty expression $^{26}$ for the latter, using the values of $q, M_{L}, d$, and $\alpha_{f}$ obtained above from $s_{0}$. Such a comparison for $[\eta]$ is shown in Figure $12 a$ and $b$, in which the dashed and solid curves refer, respectively, to the wormlike chains without and with excluded volume. These curves are shown for $\bar{M}_{w}$ higher than $10^{4}$, since below this limit Yamakawa-Fujii's theoretical values are unavailable. The theoretical solid lines, though close to the observed data, overestimate the excluded-volume effect. It is known for flexible chains with small excluded- 


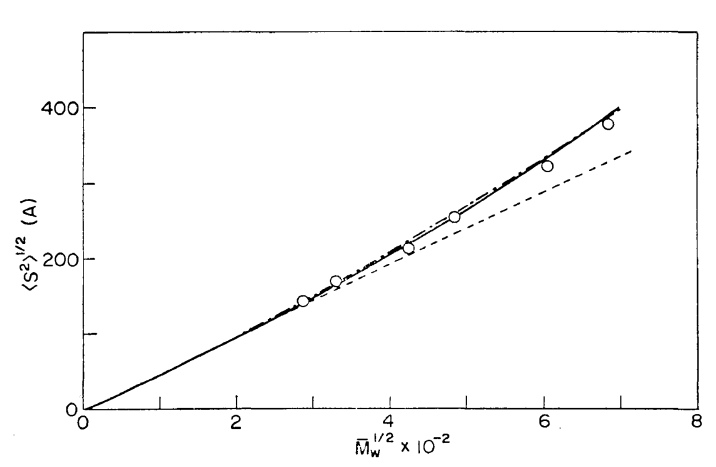

Figure 13. Comparison of $\left\langle S^{2}\right\rangle$ data for polycarbonate in THF with theoretical values. Dashed line, unperturbed $\left\langle\boldsymbol{S}^{2}\right\rangle\left(\left\langle\boldsymbol{S}^{2}\right\rangle_{0}\right)$ calculated from eq 9 with $q=18 \mathrm{~A}$ and $M_{L}=26$ daltons/A; solid line, $\alpha_{f}{ }^{2}\left\langle S^{2}\right\rangle_{0}$; chain line, $\alpha_{s}{ }^{2}\left\langle S^{2}\right\rangle_{0}$ calculated with $q=18 \mathrm{~A}, M_{L}=26$ daltons $/ \mathrm{A}$, and $B=0.05$.

volume effects that values of the viscosity-radius expansion factor $\alpha_{\eta}$ are smaller than those of $\alpha_{f}$ when compared under the same conditions. ${ }^{5}$ Part of the discrepancy between theory and experiment in Figure 12 may be accounted for by this fact. It is to be noted here that the slope of the log-log plot for the unperturbed chain decreases gradually from 0.77 to 0.55 as $\bar{M}_{w}$ increases. Figure 13 shows a comparison of our $\left\langle S^{2}\right\rangle$ data with theoretical values. The dashed line refers to the unperturbed dimensions $\left\langle S^{2}\right\rangle_{0}$ calculated from $^{26}$

$$
\begin{aligned}
\left\langle S^{2}\right\rangle_{0}= & (q L / 3)\{1-(3 q / L)[1-2(q / L) \\
& \left.\left.+2(q / L)^{2}-2(q / L)^{2} \exp (-L / q)\right]\right\}
\end{aligned}
$$

On the other hand, the solid line represents $\left\langle S^{2}\right\rangle_{0} \alpha_{f}{ }^{2}$. The agreement between the experimental data and the solid curve is satisfactory; note that for flexible chains with small excluded volumes $\alpha_{f}$ is closer to the expansion factor $\alpha_{s}$ for $\left\langle S^{2}\right\rangle$ than it is to $\alpha_{\eta}$. Thus, we find that the present experimental data for $s_{0},[\eta]$, and $\left\langle S^{2}\right\rangle$ in THF are fitted reasonably well by the wormlike chain model with the parameters given in Table III and the $\alpha_{f}$ deduced from the $s_{0}$ data.*

* The value of $q=18$ (A) gives 1.4 for the conformational parameter $\sigma$, using the fact that, if it is assumed that all carbonate groups are in the transtrans configuration and use is made of the structural data assigned by Williams and Flory, ${ }^{27}$ the length,
We finally apply the data of $A_{2}$ for the THF solutions to Yamakawa-Stockmayer's theory, ${ }^{28}$ which has been formulated for the wormlike bead in the double contact approximation and can be written

$$
A_{2}=\frac{N_{\mathrm{A}} q B}{M_{L}{ }^{2}}\left[1-(3 / 2 \pi)^{3 / 2} B Q L^{1 / 2}+\cdots\right]
$$

with the "reduced" excluded volume parameter $B$ defined by

$$
B=\beta /\left(2 q a^{2}\right)
$$

Here $Q$ is a complicated function of $q, M_{L}$ and $d$ (see ref 28), $\beta$ is the binary cluster integral between a pair of beads, and $a$ is the contour distance between two adjacent beads. Equation 10 indicates that if the third and higher terms in the brackets can be neglected in comparison to unity, a plot of $A_{2} v s$. $Q L^{1 / 2}$ should follow a straight line, and that $B$ can be evaluated from both intercept and slope. However, this method is not precisely applicable to the system polycarbonate-THF, since, when judged from the $\alpha_{f}$ in Figure 11, these higher terms may not be negligible. A rough estimate of $B$ by the use of eq 10 and the parameters in Table III gives $0.05 \pm 0.01$. Taking $a$ in eq 11 to be the contour length per monomer, this in turn gives $\beta \approx 170 \times$ $10^{-24}\left(\mathrm{~cm}^{3}\right)$, which is much larger than the values, $20-40 \times 10^{24}$, reported for typical flexible polymers in very good solvents. ${ }^{5,6}$ It is of importance that, while the $\beta$ value for polycarbonate in THF is as unusually large as this, the $\alpha_{f}$ and $\alpha_{s}$ are quite small, as can be seen from Figures 11 and 13 (see below for $\alpha_{s}$ ). This feature also can be taken as a reflection of the stiff nature of the polycarbonate chain.

With the value of $B=0.05$, we have calculated $\left\langle S^{2}\right\rangle\left(=\alpha_{s}{ }^{2}\left\langle S^{2}\right\rangle_{0}\right)$, using eq 9 for $\left\langle S^{2}\right\rangle_{0}$ and the first-order perturbation theory ${ }^{28}$ for $\alpha_{s}^{2}$, i.e.,

$\alpha_{s}{ }^{2}=1+(67 / 70) K(L / 2 q)(3 / 2 \pi)^{3 / 2} B\left(M / 2 q M_{L}\right)^{1 / 2}+\cdots$

$A(=2 q)$, of Kuhn's statistical segment for polycarbonate is given approximately by $A=18.6 \times 10^{-8}$ $\sigma^{2}(\mathrm{~cm}) .^{5}$ Williams and Flory have shown that the data of Berry, et al., for $[\eta]$ and $\left\langle S^{2}\right\rangle$ in theta solvents yield $\sigma \approx 1$. The discrepancy between this and our value of 1.4 may be attributed to a solvent effect either on $q$ or $M_{L}$. 
where, in accordance with Yamakawa and Stockmayer, ${ }^{28}$ it was assumed that, excepting the factor of $67 / 70$, the coefficient $K(L / 2 q)$ was the same as that in the expansion of the expansion factor $\alpha_{R}$ for the mean-square end-toend distance. The chain line in Figure 13 represents the results obtained. It runs quite close to the experimental points and the solid line. In summary, the wormlike bead model consistently interprets the intra- and intermolecular excluded-volume effects for polycarbonate in THF.

Acknowledgments. The authors wish to thank Professor H. Yamakawa of Kyoto University for providing the numerical tables of $s_{0}$ and $[\eta]$ and Professor G.C. Berry of Mellon University for his interest in the present work. Thanks are also due to Mr. T. Ooe, the Head of the Osaka Plant of Mitsubishi Gas Chemical Company, Ltd., and Mr. H. Yoshizaki, the Chief of its Plastics Department, who kindly prepared polymer samples for this work.

\section{REFERENCES}

1. G. V. Schulz and E. Penzel, Makromol. Chem., 112, 260 (1968).

2. E. Penzel and G. V. Schulz, ibid., 113, 64 (1968).

3. L. J. Fetters and H. Yu, Macromolecules, 4, 385 (1971).

4. M. Fujii, K. Honda, and H. Fujita, Biopolymers, 12, 1177 (1973).

5. H. Yamakawa, "Modern Theory of Polymer Solutions," Harper \& Row, New York, N.Y., 1971.

6. H. Yamakawa, Pure Appl. Chem., 31, 179 (1972).
7. G. V. Schulz and A. Horbach, Makromol. Chem., 29, 93 (1959).

8. G. Sitaramaiah, J. Polym. Sci. A, 3, 2743 (1965).

9. G. C. Berry, H. Nomura, and K. G. Mayhan, ibid., A-2, 5, 1 (1967).

10. W. R. Moore and M. A. Uddin, Europ. Polym. J., 5, 185 (1969); 6, 121 (1970).

11. H. Yamakawa, J. Chem. Phys., 45, 2606 (1966).

12. Gj. Deželič and J. Vavra, Croat. Chim. Acta, 38, 35 (1966).

13. J.P. Kratohvil, Gj. Deželič, M. Kerker, and E. Matijevič, J. Polym. Sci., 57, 59 (1962).

14. G. C. Berry, J. Chem. Phys., 44, 4550 (1966).

15. A. de Chirico, Chim. Ind. (Milan), 42, 248 (1960).

16. M. L. Huggins, J. Amer. Chem. Soc., 64, 2716 (1942).

17. D. F. Mead and R. M. Fuoss, J. Amer. Chem. Soc., 64, 277 (1942).

18. R. L. Baldwin, Biochem. J., 55, 644 (1953).

19. H. Fujita, "Foundations of Ultracentrifugal Analysis," Interscience Publishers, Inc., New York, N.Y., 1975.

20. J. E. Blair and J. W. Williams, J. Phys. Chem., 68, 161 (1964).

21. H. Murakami, T. Norisuye, and H. Fujita, Polymer J., 7, 274 (1975).

22. O. Kratky and G. Porod, Rec. Trav, Chim., 68, 1106 (1949).

23. H. Yamakawa and M. Fujii, Macromolecules, 6, 407 (1973).

24. H. Yamakawa and M. Fujii, ibid., 7, 128 (1974).

25. R. Bonart, Makromol. Chem., 92, 149 (1966).

26. H. Benoit and P. Doty, J. Phys. Chem., 57, 958 (1953).

27. A. D. Williams and P. J. Flory, J. Polym. Sci. A-2, 6, 1945 (1968).

28. H. Yamakawa and W. H. Stockmayer, J. Chem. Phys., 57, 2843 (1972). 